\section{Fortuitous oxidations by methane-utilizing bacteria}

A MAJOR thesis of Higgins et al. ${ }^{1}$ is that the wide substrate specificity shown by methane monooxygenase (MMO) has developed because of its survival value to the species by enabling the organisms to use ancillary carbon or energy sources.

In support of their thesis, the authors state that "such extraordinary lack of substrate specificity would be extremely unusual if it were entirely fortuitous". There are, however, many examples of oxygenases and other enzymes which have a wide substrate range but which are only metabolically functional for a single substrate. For example, ribulose bisphosphate carboxylase in all autotrophs has both carboxylation and oxygenation activities, the latter apparently leading to wasteful metabolism which has not been eliminated over a long period of biochemical evolution ${ }^{2}$. Nitrogenase, an enzyme which reduces many triply bonded substrates, also produces dihydrogen as an intrinsic part of the reduction process, clearly a wasteful mechanism ${ }^{3}$. If there were some survival value to the species there would be a strong selective pressure on the organism to eliminate these 'side' reactions or to retain, evolve or acquire those enzymes that were capable of assimilating the product into biomass. Although it is possible that acetate could arise as a result of $\beta$-oxidation of 1-phenylheptane and $\omega$-oxidation of higher alkanes and may therefore subsequently yield reducing equivalents, there is no direct evidence that any of the biotransformation products given in ref. 1 , Table 1 are incorporated into biomass. Indeed, the biotransformation of most of these substrates results in a net loss of available energy to the cell because they require $\mathrm{NADH}$ for oxygenation to the product.

It is possible, for example, that the oxidation of toluene to benzoic acid may result in a net gain in reducing equivalents due to the activity of nonspecific alcohol dehydrogenases; however, no NADH is generated. As MMO requires NAD(P)H in vitro ${ }^{4-6}$ (and probably in vivo also), any oxidation that does not give rise to NAD(P)H may well be detrimental to the cell's overall energy balance. Furthermore, it has been calculated that the $\mathrm{NAD}(\mathrm{P}) \mathrm{H}$-requiring MMO organisms are probably limited for $\mathrm{NAD}(\mathrm{P}) \mathrm{H}$ supply during growth on methane ${ }^{7}$. The fact that some of the products are certainly more toxic to the cell than the substrate also argues against the retention of a widespecificity MMO. We therefore contend that these biotransformations, which are supposed to aid the survival of the organisms, probably have exactly the opposite effect.

On closer examination of the paper quoted by the authors on their own work using whole cells, one observes that they were using suspensions containing 70$80 \mathrm{mg}$ dry weight of organisms per $20 \mathrm{ml}$ to effect the biotransformations of these compounds over a 15-24-h period. The high cell concentration necessary to detect oxidation is clearly unrepresentative of the situation in the biosphere.

Therefore, we feel that the arguments for the wide substrate specificity of MMO having "substantial metabolic significance" are invalid. We re-affirm our belief that all these oxidations are purely fortuitous ${ }^{8}$ and that there is no direct evidence for the oxidation of any substrate by MMO, other than methane, conferring a special advantage to the cell, whether it be as a supplementary carbon source or a provider of useful energy. We have always subscribed to the view that methanotrophs have a significant role in the oxidation of organic compounds and ammonia in the environment, but purely by a fortuitous mechanism and not one that has evolved or been retained to confer a substantial metabolic advantage to the cell.

Some methanotrophs, faced with a shortage of methane in the environment, may well oxidize any available ethane to acetate, but significant amounts of such acetate could be incorporated only by the type II methanotrophs (and not type I methanotrophs as the latter have an incomplete tricarboxylic acid cycle). Acetate could only serve as a source of carbon and not ATP because these organisms are unable to grow on acetate alone. Even so, it would still be necessary to generate NADH for ethane oxidation from the oxidation of methane to $\mathrm{CO}_{2}$. But why is it necessary to do this for survival? All the methane oxidizers (where explored) seem to be able to form resting cells (cysts or exospores) which can survive in a hypobiotic state for years. Surely the elaboration of these protective mechanisms is far more relevant to survival than a catholic MMO enzyme able to scavenge trace amounts of carbon from the environment for the doubtful pleasure of making acetate?

D. I. STIRLING
H. DALTON
Department of Biological Sciences,
University of Warwick,
Coventry CV4 7AL, UK

1. Higgins, I. J., Best, D. J. \& Hammond, R. C. Nature 286, 561-564 (1980).

2. Ellis, R. J. Trends biochem. Sci. 4, 241-244 (1980)

3. Robson, R. L. \& Postgate, J. R. A. Rev. Microbiol. 34, 183-207 (1980).

4. Colby, J., Dalton, H. \& Whittenbury, R. Biochem. J. 151, 459-462 (1975).
5. Colby, J. \& Dalton, H. Biochem. J. 157, 495-497 (1976). 6. Stirling, D. I. \& Dalton, H. Eur. J. Biochem. 96, 205-212 (1979).

7. Anthony, C. J. gen. Microbiol. 104, 91-104 (1978).

8. Stirling, D. I. \& Dalton, H. FEMS Microbiol. Lett. 5, 315-318 (1979).

HIGGins ET AL. REPLY-We do not accept the argument that there is nothing unusual about the extreme lack of specificity of some methane monooxygenases (MMO). Most oxygenases have a relatively narrow substrate range, some of them only acting on one or two very similar compounds. Even in the case of enzymes closely related to MMO, the microbial alkane hydroxylases, each one is restricted to quite a narrow band of chain lengths. The only oxygenase of which we are aware that approaches MMO in its 'catholic taste' for substrates is the mammalian liver P450 LM fraction ${ }^{1}$. Here it is' very probable that there has indeed been selection for broad specificity as this enzyme is essential to initiating processes for the removal of a wide range of toxic metabolites and xenobiotics from the body. In addition, in some methanotrophs the MMO seems to have a more restricted substrate range ${ }^{2}$, showing that extreme lack of specificity is not a mechanistic dictum.

We accept that the lack of specificity of ribulose bisphosphate carboxylase and nitrogenase leads to wasteful metabolism in some circumstances and various species have developed mechanisms to minimize this in the former case. Nevertheless, compared with MMO these are highly specific enzymes. Indeed, ribulose bisphosphate carboxylase is specific for $\mathrm{O}_{2}$ or $\mathrm{CO}_{2}$.

In reply to the specific points raised by Stirling and Dalton:

(1) It is pointed out that many of the reactions catalysed are actually detrimental to the organisms because of reducing power consumption or the generation of toxic products. This point was made in our review. If this is the case, lack of development of more specific enzymes would seem to require some explanation. In fact, of course, this is something of a 'red herring' because most compounds of that type are not present in the environment in substantial amounts. Some have only recently been introduced as a result of the activities of the organic chemist. Therefore, we agree that many of these actual oxidations are probably purely fortuitous, although the ability to carry out some of these oxidations, we suggest, has been retained as a desirable characteristic. We did not imply that most of the reactions in our Table 1 lead to utilizable carbon or energy generation. The purpose of the table was simply to give an idea of the types of reactions catalysed. Such reactions may be of industrial value and may be involved in 